\title{
Dependence of Premature Births from Periodontal Status of Pregnant Women
}

\author{
Sonila Robo ${ }^{1}$, Ilma Robo ${ }^{2}$, Eduart Kapaj ${ }^{1}$, Saimir Heta $^{3}$ \\ ${ }^{1}$ Department of Gynecology Obstetrics, Qendra Spitalore Fier, Fier, Albania \\ ${ }^{2}$ Department of Periodontology, Albanian University, Tirana, Albania \\ ${ }^{3}$ Department of Pediatric Surgery, Qendra Spitalore Universitare, Tirana, Albania
}

Email address:

ilmarobo@yahoo.com (I. Robo)

\section{To cite this article:}

Sonila Robo, Ilma Robo, Eduart Kapaj, Saimir Heta. Dependence of Premature Births from Periodontal Status of Pregnant Women. International Journal of Biomedical Engineering and Clinical Science. Vol. 4, No. 2, 2018, pp. 54-57. doi: 10.11648/j.ijbecs.20180402.14

Received: March 10, 2018; Accepted: March 28, 2018; Published: May 9, 2018

\begin{abstract}
Background: Early birth is 37 weeks or less, pregnancy maturity! Clinically active presence, or positive culture of vaginal secretions, means excessive production of cytokines and prostaglandins also encountered in amniotic fluid. Bacterial vaginosis appears with their clinical outbreak in a combination of bacteria. Some of these bacteria are basic members in the creation of bacterial plaque. Objective: The purpose of this study is to find the link between the presence of specific bacteria in the mouth, bacterial vaginosis as one of the causes of premature birth, and the latter. Methods: The study was applied to 30 pregnant women in the burden pathology ward at Fier maternity, divided into two groups. The first group consisting of 20 women in the 2-month period, August-September 2011. The number of women in the ward was 10 days maximum! All women were treated with cortisone and serum IV, magnesium sulphate, antibiotic prophylaxis! Results: $55 \%$ of women were under the age of 25 and $45 \%$ of women were over the age of 25 . The age effect is mentioned for classifying the diseases that causes Actinomyces. Under the age of 25, a teenager and a 25-year-old are chronically ill. The final index was G2! All females were positive for the presence of salicylic acid in saliva and vaginal secretions. Conclusions: Premature birth is a complex process with some gynecological reasons, but in high percentage of cases there is coverage with the presence of infection by Actinomyces Actinomycetemcomitans in the oral cavity, which depending on the age causes two different types of periodontitis with special characteristics.
\end{abstract}

Keywords: Early Birth, Periodontal Status, Bacterial Vaginosis

\section{Introduction}

In obstetric more frequent uterine contractions occur in 1 hour and cervical dilatation. In high percentage is preceded by the outbreak of vaginal infections and placental laceration in thickness. The uterus is strong, contracted and in this condition, increases the possibility of further contraction. In the presence of vaginosis, the production of prastaglandins and cytokines is increased in female genital organs, which is a common disorder in female reproductive age [1-3].

Spontaneous premature births appear for reasons and causes before pregnancy: the age of early or late pregnancy, previous abortions, lack of vitamins, obesity - the amount of cholesterol. The presence of Actinomyces as a gramnegative, anaerobic, kapnofil, a major pathogenic agent of periodontal disease, is associated with the appearance of specific Ig's in the blood. This bacterium serves as a source of infection and rupture of placental membranes in hematological pathways and direct pathways [3]. Bacterial vaginosis is caused by candida, lactobacilli, gardinelat, prevotela, fusobacterium nucleatum, gingivalis porphiromonas. Associative elements, albeit in low percentage, are fusobakterium nucleatum and gingival porphiromonas. Fusobakterium Nucleatum produces butyral acid, similar to Clostridium, but unlike the latter, is found in oral cavity [4]. Premature babies with low infant weights have a positive culture even in the absence of clinically visible infection.

Fusobacterium Nucleatum are gram-negative fusioform rods, do not produce spores, are not motile [4].

Adhere to a large number of gram-positive bacteria, gram- 
negative (Porphiromonas Gingivalis) and viruses. Elements that differentiate this bacterium from other gram-negative, non-motile bacteria that do not produce spores are as follows [4-6]:

a. Produces butyric acid as the result of glucose fermentation and peptone.

b. Use amino acids, glutamine, histidine, aspartate to produce cellular energy.

c. Glucose is mainly used for production of cellular molecules.

d. It lives in the environment with $6 \%$ oxygen solubility.

Porphiromonas gingivalis, the other bacterium of interest in this paper, has these specific elements $(3,7,8)$ :

a. Living in symbiosis with pallidum treponema and forsithus bacterium.

b. It is preferable to enter the gingival sulcus.

c. Produces proteolytic proteins that destroy cysteine and arginine.

d. The main target of these proteins are the fibers of the periodontal ligament.

The purpose of this study is to find the interconnection between the presence of specific bacteria in the mouth and bacterial vaginosis, as the latter is one of the causes of premature births. In the assessment are received specific presence of fusobacterium nucleatum, porphiromonas gingivalis and actinomyces actinomycetemcomitans. Gingival status analyzes were performed based on the periodontal morbidity index - PDI, on specific teeth according to the Ramfjord index [3].

\section{Methods}

The study was applied to 30 pregnant women in the burden pathology ward at Fier maternity, divided into two groups. The first group consisting of 20 women in the 2-month period, August-September 2011. The number of women in the ward was 10 days maximum! All women were treated with cortisone and serum IV, magnesium sulphate, antibiotic prophylaxis!

The following analyzes were performed on each female:

a. microbial culture of material obtained from gingival sulcus,

b. Ig specifics of plasma,

c. fibronectin test in cervical secretions

d. quantitative analysis of the sulcular Ig.

The second group of 10 pregnant women, with a positive culture of vaginal secretions, are presented in the period December-January 2017 and subject to these analyzes:

a. selective cultivation of vaginal secretions, b. amniocentesis - detection of prostaglandin E2 - PGE2 with parental preference,

c. detection of butyric acid in vaginal secretions,

d. gingival index assessment (gingival index - teeth according to Ramfjord - PDI): 16, 21, 24, 36, 41, 44 assessment of the degree of gingival inflammation as follows:

G0 = lack of inflammation

$\mathrm{G} 1$ = gingival inflammatory changes from simple, to moderate, not completely spread to the analyzed tooth.

$\mathrm{G} 2$ = light to moderate gingivitis, extending into the complete tooth.

G3 = engraved gingivitis, characterized by bleeding, apparent tendency to bleeding and ulceration [3].

\section{Results}

In the first group:

12 females were infected with Actinomyces Actinomycetemcomitans, as confirmed by specific Ig assays.

a. 4 women suffered premature birth due to vaginal infections.

b. 7 females suffered premature birth as a result of premature rupture of the membranes.

c. 9 females of premature spontaneous birth (without noticeable cause).

d. 11 women were under the age of 25 and 9 women over the age of 25 .

$20 \%$ of women suffered from vaginal infections (mycoplasma, streptococcus beta hemolytic, trichomos vaginalis).

$35 \%$ of women with premature ruptured membranes, were positive with test of fibronectin. Premature rupture of the membranes may be caused by causes during pregnancy (reasons why women are exposed in the burden pathology ward): high arterial hypertension, cervical constipation, abnormal fluid amniotic quantities, smoking, alcohol, cocaine, stress and body fatigue, high fetal endocrine activity, excessive uterine tension, decay bleeding, etc. [2]. This element is captured by the fibronectin test.

a. $45 \%$ of women suffered premature spontaneous birth, due to reasons during pregnancy.

b. $60 \%$ of women were infected with Actinomyces Actinomycetemcomitans and were suffering from active periodontitis.

Table 1 summarizes the data recorded for the first group of patients, coupled with the respective age of the patient and the age of pregnancy.

Table 1. Data collected after analyzing the first group of patients under study.

\begin{tabular}{|c|c|c|c|c|c|c|}
\hline Patients 1 & Age 28 & Week of Pregnancy 36 & Ig blood positive & Fibronectin positive & infections positive & Ig salivar pozitive \\
\hline 2 & 32 & 36 & - & & & - \\
\hline 3 & 25 & 35 & & & - & - \\
\hline 4 & 25 & 35 & - & - & & - \\
\hline 5 & 18 & 36 & - & - & & - \\
\hline 6 & 27 & 32 & - & & - & - \\
\hline 7 & 23 & 27 & - & & & \\
\hline
\end{tabular}




\begin{tabular}{|c|c|c|c|c|c|c|}
\hline Patients 1 & Age 28 & Week of Pregnancy 36 & Ig blood positive & Fibronectin positive & infections positive & Ig salivar pozitive \\
\hline 8 & 37 & 34 & & & & \\
\hline 9 & 20 & 37 & - & - & & - \\
\hline 10 & 18 & 36 & & & - & - \\
\hline 11 & 30 & 36 & - & & & - \\
\hline 12 & 22 & 37 & - & - & & - \\
\hline 13 & 34 & 35 & & & - & \\
\hline 14 & 26 & 36 & - & - & & - \\
\hline 15 & 23 & 36 & & & & - \\
\hline 16 & 26 & 36 & - & - & & \\
\hline 17 & 21 & 36 & - & & & - \\
\hline 18 & 21 & 36 & - & - & & \\
\hline 19 & 23 & 35 & & & & \\
\hline 20 & 25 & 37 & & & & \\
\hline
\end{tabular}

$55 \%$ of women were under the age of 25 and $45 \%$ of women were over the age of 25 . The age effect is mentioned for classifying the diseases that causes Actinomyces. Under the age of 25, a teenager and a 25-year-old are chronically ill.

For the second group, the following results were obtained:

a. Of the 10 pregnant women, six of them were born prematurely with low infant weight. Infant weights ranged from 2020 to $2700 \mathrm{gr}$.

b. 4 were clinically infected with candida-induced vaginosis. c. 2 females (the only ones who received amniocentesis) were positive against PGE2 and were with clinically active vaginosis.

All females were positive for the presence of salicylic acid in saliva and vaginal secretions.

Table 2 shows periodontal indexes in patients of the second study group.

Periodontal index in patients under study, according to selected groups.

Table 2. Periodontal indexes at patients of the second group of the study.

\begin{tabular}{lllllll}
\hline Nr. & $\mathbf{1 6}$ & $\mathbf{2 1}$ & $\mathbf{2 4}$ & $\mathbf{3 6}$ & $\mathbf{4 1}$ & $\mathbf{4 4}$ \\
\hline Patient 1 & G0G1G0 & G1G1G1 & G1G2G1 & G2G2G3 & G2G0G2 & G0G0G1 \\
Patient 2 & G3G2G3 & G0G0G0 & G2G1G2 & G2G2G2 & G0G0G1 & G3G3G3 \\
Patient 3 & G3G3G3 & G2G2G2 & G3G3G3 & G3G3G3 & G3G3G3 & G3G3G3 \\
Patient 4 & G0G1G1 & G0G0G0 & G0G0G0 & G3G3G3 & G2G3G2 & G2G2G2 \\
Patient 5 & - & G2G1G2 & G0G0G0 & - & G1G1G1 & G0G0G0 \\
Patient 6 & G2G2G2 & G2G2G2 & G3G3G3 & G2G2G3 & G1G0G1 & G2G2G2 \\
\hline
\end{tabular}

The PDI index, based on the average of the indexes collected is as follows:

Patient 1 (Go, G1, G1, G2, G2, G0) -G1

Patient 2 (G3, G0, G2, G2, G0, G3) -G1

Patient 3 (G3, G3, G3, G3, G2, G3) -G3

Patient 4 (G1, G0, G0, G3, G2, G2) -G1

Patient 5 (G2, G0, G1, G0) -G1

Patient 6 (G2, G2, G3, G3, G1, G2) -G2.

The final index was G2!

Table 3 presents the data of six patients with low infant weights. These are the same patients in which the selected periodontal dental index was evaluated.

Table 3. Patient Ranking according to Card Data.

\begin{tabular}{llll}
\hline No. & Age of the patient & Age of pregnancy & Infant Weight \\
\hline Patient 1 & 25 & 34 & 2200 \\
Patient 2 & 30 & 37 & 2600 \\
Patient 3 & 21 & 36 & 2650 \\
Patient 4 & 23 & 27 & 2020 \\
Patient 5 & 23 & 34 & 2400 \\
Patient 6 & 25 & 37 & 2500 \\
\hline
\end{tabular}

Based on literature, Periodontal disease in pregnant women with a reservoir of organisms and their products can be considered a risk factor for adverse pregnancy outcome (9). The periodontal disease as a probable risk for adverse pregnancy outcomes such as preterm delivery and low birth weight, so, health workers should be encouraged to promote good oral health among women (10). A fetal fibronectin test may be of additional value in the prediction of preterm birth. The most effective methods to prevent preterm birth depend on the obstetric history, which makes the identification of women at risk of preterm birth an important task for clinical care providers [11].

\section{Conclusions}

The study is on track of the data received at this department in the February-March period 2011. Data have interesting variability limits. Premature birth is a complex process with some gynecological reasons, but in high percentage of cases there is coverage with the presence of infection by A. Actinomycetemcomitans in the oral cavity, which depending on the age causes two different types of periodontitis with special characteristics.

For the second group of women involved in the study: Bacterial vaginosis is a phenomenon that causes about $1 / 5$ of premature births. The presence of positive, clinically active, vaginal secretion culture means excessive production of cytokines and prostaglandins encountered in amniotic fluid.

The beginnings of pregnancy are associated with gingival dysfunction due to infections and hormone fluctuations. The G2 index speaks of hormonal effects and serves as an 
indicator of gingival inflammation.

Fusobakterium Nukleatum and Porphiromonas Gingivalis are about $13 \%$ of the component of vaginal secretion culture in bacterial vaginosis. The prevalence of these bacteria in premature ejaculate women is distinct, but rarely occurs in women without bacterial vaginosis.

\section{References}

[1] Martinho FC, Chiesa WM, Leite FR, Cirelli JA, Gomes BP; "Antigenicity of primary endodontic infection against macrophages by the levels of PGE (2) production"; 37 (5):602-7. Epub 2011 Mar 5.

[2] Jia Xu, "Bacterial Vaginosis, Vaginal Fluid Defensins, and Preterm Delivery"; Michigan State University, 2007, 49 pages.

[3] Newman, Takey, Caranzas; "Clinical Periodontology", edicioni i nëntë, viti i botimit 2004.

[4] Bolstad, A. I., Jensen, H. B., Bakken, V. "Taxonomy, Biology and Periodontal Aspects of Fusobacterium nucleatum." Clinical of Microbiology Reviews. Jan. 1996. pp. 55-71.

[5] Gemmell, E., Bird, P. S., Carter, C. L., Shenker, B. J. "Identification and analysis of fipA, a Fusobacterium nucleatum immunosuppressive factor gene." Infect Immun. April 1996. 1335-1341.

[6] Silva, V. L., Diniz C. G., Santos, S. G., Nicoli J. R., Carvalho, M. A., Farias, L. M. "Enhancad pathogenicity of Fusobacterium nucleatum adpted to oxidative stress." Microbial Pathogenesis. Oct. 2005. pp. 131-138.

[7] P. I. Diaz, P. S. Zilm and A. H. Rogers "Fusobacterium nucleatum supports the growth of Porphyromonas gingivalis in oxygenated and carbon-dioxide-depleted environments"; Microbiology (2002), 148, 467-472.

[8] Gaffey JD, Rogers AH Gully NJ. 1997. Protease production in Porphyromonas gingivalis grown under different conditions. Aust. Dent. J. 42, 277-8.

[9] Meqa K, Dragidella F, Disha M, Sllamniku-Dalipi Z, "The Association between Periodontal Disease and Preterm Low Birthweight in Kosovo" Acta Stomatol Croat. 2017 Mar; 51 (1):33-40. doi: 10.15644/asc51/1/4 .

[10] Soroye M, Ayanbadejo P, Savage K, Oluwole A.; "Association between periodontal disease and pregnancy outcomes"; Odontostomatol Trop. 2015 Dec; 38 (152):5-16.

[11] Koullali B, Oudijk MA, Nijman TA, Mol BW, Pajkrt E; "Risk assessment and management to prevent preterm birth" Semin Fetal Neonatal Med. 2016 Apr; 21 (2):80-8. doi: 10.1016/j.siny.2016.01.005. Epub 2016 Feb 18. 